\title{
Variabilidade espacial da resistência do solo à penetração em um sistema de semeadura direta
}

\author{
Spatial variability of the soil resistance to penetration in a no-tillage system
}

\section{Jorge Wilson CORTEZ ${ }^{1}$; Carlos Thiago Mercado MORENO²; Luan Santos FARINHA ${ }^{3}$; Salvio Napoleão Soares ARCOVERDE ${ }^{4}$; Igor Queiroz Morães VALENTE ${ }^{5}$}

1 Eng. Agrônomo, Doutor, Professor, Faculdade de Ciências Agrárias, Universidade Federal da Grande Dourados -
UFGD, Rodovia Dourados-Itahum, km 12, Cidade Universitária, Dourados, MS. CEP: 79804-970,
jorgecortez@ufgd.edu.br
2 Eng. Agrícola, Universidade Federal da Grande Dourados - UFGD, carlos_myjago@hotmail.com
${ }^{3}$ Eng. Agrícola, Universidade Federal da Grande Dourados - UFGD, luan_farinha10@hotmail.com
4 Eng. Agrícola e Ambiental, Pós-Doutorando, Universidade Federal da Grande Dourados - UFGD.
salvionapoleao@gmail.com
5 Eng. Agrícola, Mestre, Doutorando, Universidade Estadual de Campinas - UNICAMP, Igor_valente88@hotmail.com

Recebido em: 10-09-2018; Aceito em: 14-02-2019

\begin{abstract}
Resumo
A compactação do solo reduz a qualidade estrutural do solo e pode limitar a produção agrícola, devendo ser monitorada periodicamente. A resistência do solo à penetração tem sido utilizada por ser um indicador prático e por estabelecer relação com a densidade e o teor de água no solo. Objetivou-se avaliar a variabilidade espacial da resistência à penetração $(\mathrm{RP})$ de um Latossolo Vermelho distroférrico em sistema de semeadura direta. A coleta de dados ocorreu em área experimental de 10,54 ha, utilizando-se de grade amostral composta de 43 pontos. Em cada ponto, foi determinada a RP a partir de três medições, a partir das quais foi obtida a RP média de cada camada, a RP máxima e a profundidade da RP máxima na camada de 0-0,40 m perfil. Amostras com estrutura deformada foram coletadas de forma aleatória na área, para a determinação do teor de água no solo (U). Ocorreu dependência espacial para RP dentro da grade amostral. Valores intermediários (2,00-3,00 MPa) e críticos (3,00-4,00 MPa) de RP foram verificados em grande parte da área. Houve aumento da RP com a profundidade, atingindo níveis máximos principalmente entre 0,20 e 0,25 m. A geoestatística permitiu o zoneamento da área definindo regiões com níveis críticos de compactação, auxiliando na tomada de decisões quanto a procedimentos de manejo de solo.
\end{abstract}

Palavras-chave adicionais: agricultura de precisão; geoestatística; mecanização agrícola.

\begin{abstract}
Soil compaction reduces soil structural quality and may limit agricultural production and should be monitored periodically. The soil resistance to penetration has been used as a practical indicator and to establish relation with the density and water content in the soil. The objective of this study was to evaluate the spatial variability of the penetration resistance of an Oxisol. The data collection took place in an experimental area of $10.54 \mathrm{ha}$, using a sampling grid composed of 43 points. RP were determined from three measurements, from which the mean RP, the maximum RP, and the depth of the maximum RP in the 0-0.40 m layer were determined. Disturbed samples were collected at random in the area for determining soil water content $(\mathrm{U})$. All the variables under study presented spatial dependence within the sampling grid. Intermediate (2.00-3.00 MPa) and critical (3.00-4.00 MPa) values of soil resistance to penetration were verified in much of the area. There was an increase in soil resistance to penetration with depth, reaching maximum levels mainly between 0.20 and $0.25 \mathrm{~m}$. Geostatistics allowed the zoning of the area defining regions with critical levels of compaction, assisting in decision making regarding the procedures for soil management
\end{abstract}

Additional keywords: agricultural mechanization; geostatistics; precision agriculture.

\section{Introdução}

Em um sistema produtivo, além dos cuidados com a fertilidade do solo, devem-se buscar condições físicas do solo adequadas às plantas durante a fase de implantação e de desenvolvimento de uma determi- nada cultura, visto que estão relacionadas à produtividade agrícola. Uma das formas de alterar a condição física do solo é por meio de seu manejo, buscando criar condições estruturais favoráveis ao crescimento e ao desenvolvimento da planta, tanto da parte aérea quanto das raízes (Bottega et al., 2011). 
Nesse sentido, a implantação de sistemas de manejo conservacionistas, como o plantio direto, traz inúmeros benefícios no tocante à conservação dos recursos naturais, por priorizar a manutenção de resíduos vegetais na superfície e por reduzir a movimentação do solo, reduzindo a erosão e aumentando a atividade biológica dos microrganismos, refletindo-se positivamente na qualidade do solo e na rentabilidade da atividade agrícola.

Apesar disso, o cultivo em plantio direto, por não adotar o revolvimento do solo, pode resultar na formação de camadas compactadas. Aliado ao mínimo revolvimento, que normalmente é realizado apenas na linha de semeadura, o intenso número de operações mecanizadas também é responsável pela formação de camadas compactadas superficiais, especialmente quando há contínuo tráfego de máquinas em conteúdos inadequados de água no solo (Streck et al., 2004), afetando a qualidade do solo e a produção agrícola (Bergamin et al., 2010a; Bergamin et al., 2010b).

O solo, em condições ideais para o desenvolvimento radicular, é explorado de forma homogênea pelas raízes das plantas, sendo o volume de solo explorado relativamente maior que em solos com problemas de compactação (Valadão et al., 2015). Isso porque determinados estados de compactação provocam modificações na estrutura do solo, acarretando aumento nos valores de densidade do solo e resistência à penetração, bem como redução da porosidade total e macroporosidade do solo (Freddi et al., 2007; Bergamin et al., 2010a; Bergamin et al., 2010b; Valadão et al., 2015).

Um dos parâmetros mais utilizados para avaliar a intensidade da compactação do solo é sua resistência à penetração, relacionada a diversos atributos do solo, indicadores do grau de compactação, que influenciam o comportamento do solo submetido a pressões externas (Oliveira Filho et al., 2015). Por ser um parâmetro prático e completo, também relacionado à textura, à densidade e ao teor de água no solo, tem sido utilizado para o monitoramento periódico do estado de compactação do solo, e como indicador na avaliação dos efeitos dos sistemas de manejo do solo sobre o ambiente radicular (Bergamin et al., 2010a; Bergamin et al., 2010b; Valadão et al., 2015).

Valores críticos de resistência do solo à penetração podem variar de 1,5 MPa a 4,0 Mpa (Rosolem et al., 1999); embora, de maneira geral, valores próximos a 2 MPa são aceitos como impeditivos ao crescimento radicular. Também têm sido observados valores de resistência do solo à penetração, críticos para o desenvolvimento radicular em sistema plantio direto de 3,0 a 4,0 MPa (Vaz et al., 2011; Betioli Júnior et al., 2012; Guimarães et al., 2013). Porém, Marasca et al. (2011), ao avaliaram a variabilidade espacial da resistência à penetração em um cultivo de soja em sistema plantio direto, verificaram valores de resistência do solo à penetração de 2,9 a 4,28 MPa, que não foram considerados restritivos à produção da cultura.

A análise do comportamento espacial dos atributos físicos do solo por meio da geoestatística permite detectar a variabilidade e distribuição espacial dos atributos estudados e, com isso, avaliar e descrever de maneira detalhada a variabilidade dos atributos do solo (Alves et al., 2014), sendo importante no manejo e no controle de fatores de produção das culturas, bem como no monitoramento ambiental (Oliveira et al., 2013).

Assim, o objetivo deste trabalho foi avaliar a variabilidade espacial da resistência à penetração de um Latossolo Vermelho distroférrico em área de semeadura direta de soja e milho em sucessão, por 20 anos.

\section{Material e métodos}

O trabalho foi desenvolvido na Fazenda Experimental de Ciências Agrárias da Universidade Federal da Grande Dourados (UFGD), localizada no município de Dourados - MS, entre as coordenadas geográficas de $22^{\circ} 14^{\prime}$ de latitude sul e 54⒌' de longitude oeste; com altitude média de $434 \mathrm{~m}$. O clima é do tipo AW, segundo a classificação de Köppen. O solo da área é um Latossolo Vermelho distroférrico, cuja granulometria está apresentada na Tabela 1.

Tabela 1 - Granulometria do Latossolo Vermelho distroférrico da Fazenda Experimental da UFGD. Granulometry of the Dystroferric Red Latosol of the Experimental Farm of the UFGD.

\begin{tabular}{lccc}
\hline Camadas $(\mathrm{m})$ & Argila & Silte $\left(\mathrm{g} \mathrm{kg}^{-1}\right)$ & Areia \\
\hline $0,00-0,10$ & 597,8 & 217,3 & 184,9 \\
$0,10-0,20$ & 592,6 & 222,4 & 185,0 \\
$0,20-0,30$ & 623,1 & 197,9 & 179,0 \\
$0,30-0,40$ & 628,3 & 202,3 & 169,4 \\
\hline
\end{tabular}

$\mathrm{Na}$ área experimental, cultiva-se como cultura de verão a soja (Glycine max), e na de inverno, o milho (Zea mays), em um sistema de sucessão de culturas sem revolvimento do solo, no qual o sistema de semeadura sobre a palha vem sendo adotado por mais de 20 anos. A área possui 10,54 ha e foi dividida numa malha com 43 pontos amostrais, utilizando-se de um sistema de navegação que realizou o contorno da área, gerando uma malha amostral com diferença entre pontos de 0,20 ha (aproximadamente $45 \mathrm{~m}$ entre pontos).

Em setembro de 2016, foi realizada a semeadura da soja na área experimental, em seguida procedeu-se à coleta de dados de resistência do solo à 
penetração $(\mathrm{RP})$, juntamente com a coleta de amostras deformadas para a determinação do teor de água no solo (U), pelo método gravimétrico. Tais amostras foram coletadas de forma aleatória na área, nas camadas de $0,00-0,10 ; \quad 0,10-0,20 ; \quad 0,20-0,30$ e 0,30-0,40 m.

Realizou-se a determinação da resistência do solo à penetração (RP) por meio de um penetrômetro eletrônico com coleta da RP a cada $1 \mathrm{~cm}$, até à profundidade de $0,40 \mathrm{~m}$, estratificada nas camadas $(0,00$ $-0,05 ; 0,05-0,10 ; 0,10-0,15 ; 0,15-0,20 ; 0,20-0,25 ; 0,25-$ $-0,30 ; 0,30-0,35$ e $0,35-0,40 \mathrm{~m}$ ) e apresentados seus respectivos valores médios de RP médio, a partir dos quais foi obtida a RP média do perfil $0,00-0,40 \mathrm{~m}$, a RP máxima do perfil $0,00-0,40 \mathrm{~m}$ e a profundidade da $\mathrm{RP}$ máxima para cada ponto.

A determinação da RP foi realizada em cada ponto amostral, onde foram realizadas três medições em um raio de até $5 \mathrm{~m}$ do ponto marcado para coleta no sistema. As coletas do $U$ e RP foram obtidas simultaneamente na fase de campo.

Os dados de RP foram analisados por meio da estatística descritiva, para permitir a visualização geral do comportamento dos dados, além de verificada a normalidade dos mesmos, utilizando o teste de Anderson-Darling. Realizou-se a análise geoestatística para verificar a dependência espacial da RP pelo cálculo da semivariância e do ajuste dos dados ao semivariograma. Os semivariogramas foram ajustados e a partir deles foram selecionados modelos com base no melhor coeficiente de determinação $\left(r^{2}\right)$ e na menor soma de quadrados do resíduo, sendo testados os modelos esférico, exponencial, gaussiano, linear, linear sem patamar e efeito pepita puro. Nos semivariogramas ajustados, foram definidos os seguintes parâmetros: efeito pepita (C0); patamar (C0 + C1) e alcance (A) (Marasca et al., 2011; Bottega et al., 2011; Bottega et al., 2013; Nagahama et al., 2016).

Posteriormente, realizou-se a interpolação por krigagem ordinária, sendo esta uma técnica de interpolação para estimativa de valores de uma propriedade em locais não amostrados. Por meio da interpolação por krigagem, os mapas de isolinhas (bidimensionais) foram construídos para o detalhamento espacial dos dados coletados, utilizando uma grade de interpolação de $20 \mathrm{~m}$.

\section{Resultados e discussão}

Os valores do teor de água no solo $(U)$, nas camadas de 0,10-0,20; 0,20-0,30 e 0,30-0,40 m (Tabela 2), encontram-se acima da faixa friável, ou seja, superior à recomendada para operações mecanizadas, sejam elas de plantio, manejo ou colheita de culturas, conforme Bottega et al. (2011). Observa-se que houve aumento do $\mathrm{U}$ em profundidade, com maior variação dos valores nas camadas superficiais e me- nor nas camadas subjacentes. Este resultado concorda com os encontrados por Bergamin et al. (2010a), estudando em condições edafoclimáticas e de manejo semelhantes.

Tabela 2 - Teor de água (U) do solo no momento da coleta da resistência do solo à penetração $(\mathrm{RP})$. Soil water contente at the time of collection of soil penetration resistance $(\mathrm{RP})$.

\begin{tabular}{cc}
\hline Camada $(\mathrm{m})$ & $\mathrm{U}(\%)$ \\
\hline $0,00-0,10$ & 13,45 \\
$0,10-0,20$ & 17,88 \\
$0,20-0,30$ & 20,65 \\
$0,30-0,40$ & 20,88 \\
\hline
\end{tabular}

Além disso, essa elevada variação do $U$ pode ser explicada pela extensão da área (10,54 ha). Bottega et al. (2011), ao avaliarem a variabilidade espacial da resistência de um Latossolo Vermelho distroférrico em área de 5,02 ha sob plantio direto, verificaram variação de $5 \%$ nos valores de U na camada de 0,00-0,20 m. Ressaltase que outros fatores, como época de amostragem, podem influenciar tal variação, assim como os valores de RP.

No resumo da análise estatística descritiva para a resistência do solo à penetração $(R P)$, nas diferentes camadas estudadas (Tabela 3), observase aumento da RP até a camada de 0,20-0,25 m, faixa onde se encontram os níveis máximos de compactação. Cortez et al. (2014) destacam que a menor RP encontrada na camada de 0,00-0,10 m, em relação às camadas subjacentes, pode ser atribuída à deposição de matéria orgânica, que contribui para a redução da densidade do solo.

A média da RP apresenta-se de baixa a intermediária, em relação ao valor crítico de 3,00 a 4,00 MPa (Vaz et al., 2011; Betioli Júnior et al., 2012; Guimarães et al., 2013); embora, segundo Rosolem et al. (1999), valores restritivos podem variar de 1,5 a 4,0 MPa em função da cultura. Marasca et al. (2011), no entanto, ao avaliarem a variabilidade espacial da RP de um Latossolo Vermelho distroférrico e sua relação com a produção de soja em sistema plantio direto, encontraram valores de RP da ordem de 2,9 a $4,28 \mathrm{MPa}$, que não se mostraram limitantes à produção da cultura.

Ainda na Tabela 3 , observa-se variação nos valores mínimos e máximos de $\mathrm{RP}$ em todas as camadas analisadas, 1,70 e 4,88 $\mathrm{MPa}$, respectivamente, demostrando que o tráfego inadequado, possivelmente, influenciou tal variabilidade do atributo. Os resultados demonstram que, apesar da grande extensão da área experimental, a variação de RP pode ser considerada homogênea.

Vale ressaltar que os valores de desvio padrão e variância (Tabela 3) foram menores na camada de 0,00-0,05 m que nas demais, demons- 
trando que, nesta camada, a variabilidade do grau de compactação é menor, mostrando que o efeito da compactação pelo tráfego de máquinas é refletido nas camadas subjacentes, ao contrário do encontrado por Bottega et al. (2011) em condições semelhantes às do presente estudo.

O teste de Anderson-Darling indicou distribui- ção normal para as variáveis analisadas. Além disso, todas as variáveis apresentaram valores de assimetria e curtose dentro do intervalo de -2 e 2 (Tabela 3), indicando que os dados respeitam uma distribuição normal (Albiero et al., 2012; Melo et al., 2013; Silva et al., 2013).

Tabela 3 - Estatística descritiva dos dados de resistência do solo à penetração (MPa). Descriptive statistics of data of soil penetration resistance (MPa).

\begin{tabular}{ccccccccc}
\hline $\begin{array}{c}\text { Camada } \\
(\mathrm{m})\end{array}$ & \multicolumn{7}{c}{ Parâmetros } \\
\cline { 2 - 9 } & Média & DP & V & Min & Max & \multicolumn{1}{c}{ A } & \multicolumn{1}{c}{ K } & AD \\
\hline $0,00-0,05$ & 0,5306 & 0,2865 & 82122 & 0,1837 & 1,2230 & 0,59 & $-0,56$ & $0,01^{* *}$ \\
$0,05-0,10$ & 1,7943 & 0,6615 & 437535 & 0,8077 & 3,0953 & 0,37 & $-1,21$ & $0,01^{* *}$ \\
$0,10-0,15$ & 2,3532 & 0,4622 & 213610 & 1,5577 & 3,6950 & 0,93 & 1,51 & $0,01^{* *}$ \\
$0,15-0,20$ & 2,6797 & 0,5385 & 289929 & 1,8290 & 4,2073 & 0,95 & 0,66 & $0,02^{* *}$ \\
$0,20-0,25$ & 2,7600 & 0,6553 & 429473 & 1,6917 & 4,8817 & 1,23 & 1,96 & $0,01^{* *}$ \\
$0,25-0,30$ & 2,6392 & 0,6958 & 484071 & 1,7040 & 4,5820 & 1,27 & 0,96 & $0,01^{* *}$ \\
$0,30-0,35$ & 2,4412 & 0,7262 & 527400 & 1,6577 & 4,4370 & 1,46 & 1,31 & $0,01^{* *}$ \\
$0,35-0,40$ & 2,2996 & 0,7183 & 515884 & 1,5497 & 4,5040 & 1,47 & 1,41 & $0,01^{* *}$ \\
$0,00-0,40$ & 2,1468 & 0,4451 & 198619 & 1,5497 & 3,4897 & 1,18 & 1,21 & $0,01^{* *}$ \\
PM & 3,3092 & 0,6911 & 477622 & 2,4230 & 5,3700 & 1,04 & 0,80 & $0,01^{* *}$ \\
Prof PM & 0,0225 & 0,0055 & 30,26 & 0,0123 & 0,036 & 0,45 & 0,15 & $0,30^{*}$ \\
\hline
\end{tabular}

${ }^{*} \mathrm{p} \geq 0,05$ dados normais, não significativo - simétrico; ${ }^{* *} \mathrm{p}<0,05$ dados não normais, significativo - assimétrico. DP: desvio padrão (MPa); V: variância; CV: coeficiente de variação (\%); Mín: mínimo (MPa); Máx: máximo (MPa); A: assimetria; K: curtose; AD: teste de Anderson-Darling. PM: Pressão máxima. Prof PM: Profundidade da pressão máxima.

A partir da análise do semivariograma para RP (Tabela 4), observou-se o ajuste do modelo esférico para as camadas avaliadas e para a análise das resistências máximas (PM) e das profundidades de ocorrência das resistências máximas (Prof PM) ao longo da área avaliada, concordando com Cortez et al.
(2014), quando analisaram RP em Argissolo Amarelo sob diferentes intensidades de tráfego de tratores. Do mesmo modo, Marasca et al. (2011) e Nagahama et al. (2016), ao avaliarem variabilidade espacial de RP, constataram maior ajuste do modelo esférico para atributos físicos do solo.

Tabela 4 - Dados ajustados do semivariograma para resistência do solo à penetração. Data ajusted of semivariogram for soil penetration resistance.

\begin{tabular}{cccccc}
\hline $\begin{array}{c}\text { Camadas } \\
(\mathrm{m})\end{array}$ & Modelo & Co & Co+C & $\mathrm{A}(\mathrm{m})$ & $\begin{array}{c}\text { Grade de } \\
\text { interpolação }\end{array}$ \\
\hline $0,00-0,05$ & Esférico & 0,00 & 119618,85 & 490,39 & 20,00 \\
$0,05-0,10$ & Esférico & 0,00 & 541593,89 & 453,60 & 20,00 \\
$0,10-0,15$ & Esférico & 0,00 & 299766,97 & 460,82 & 20,00 \\
$0,15-0,20$ & Esférico & 0,00 & 507138,73 & 630,86 & 20,00 \\
$0,20-0,25$ & Esférico & 0,00 & 1101590,48 & 966,66 & 20,00 \\
$0,25-0,30$ & Esférico & 0,00 & 1346836,16 & 1208,72 & 20,00 \\
$0,30-0,35$ & Esférico & 0,00 & 1456734,93 & 1227,99 & 20,00 \\
$0,35-0,40$ & Esférico & 0,00 & 944556,48 & 757,07 & 20,00 \\
$0,00-0,40$ & Esférico & 0,00 & 379475,11 & 680,21 & 20,00 \\
PM & Esférico & 0,00 & 969593,52 & 781,38 & 20,00 \\
Prof PM & Esférico & 0,00 & 45,68 & 501,67 & 20,00 \\
\hline
\end{tabular}

Co: efeito pepita; Co+C: patamar; A: alcance; PM: Pressão máxima. Prof PM: Profundidade da pressão máxima.

Observa-se que o efeito pepita apresentou valor zero para todos os atributos nas camadas de solos. Conforme relatado por Nagahama et al. (2016), a existência de valores de efeito pepita próximos a zero aumenta a exatidão de estimativas por meio da krigagem. Ressalta-se que, quanto menor for a pro- porção do efeito pepita em relação ao patamar do variograma, maior será a continuidade do fenômeno e a variância da estimativa, e maior será a confiança que se pode ter na estimativa (Bottega et al., 2011; Bottega et al., 2013).

Quanto aos alcances obtidos, verifica-se uma 
variação de 453,60 e 1.227,99 m, demonstrando que a malha amostral poderia ser de, no mínimo, 453,60 m de distância horizontal, uma vez que foi utilizada a distância de 45 m; ou seja, para a obtenção da mesma precisão dos dados amostrados, as malhas estudadas poderiam ter sido de, no mínimo, 453,60 m e, no máximo, de 1.227,99 m de distância entre os pontos horizontais amostrados ao longo da área experimental. Esses valores, portanto, poderiam torna-se referência em futuras coletas de RP nas condições em que se deu o estudo.

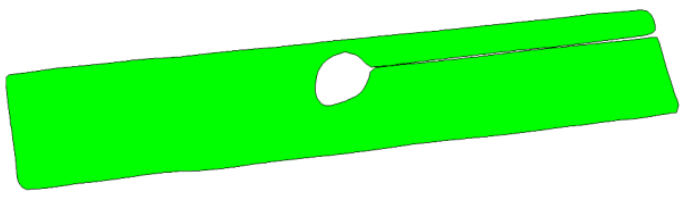

(A) $0-0,05 \mathrm{~m}$

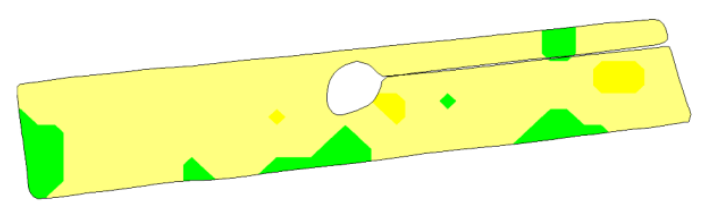

(C) $0,10-0,15 \mathrm{~m}$

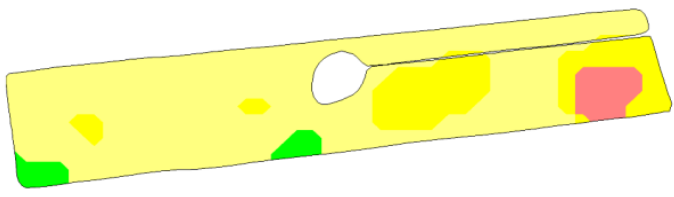

(E) $0,20-0,25 \mathrm{~m}$

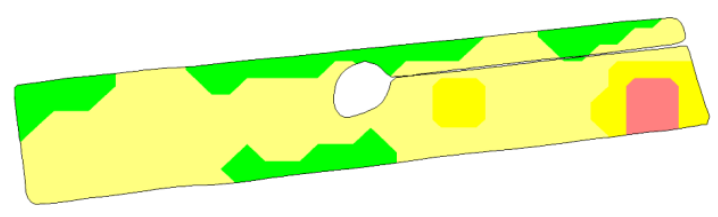

(G) $0,30-0,35 \mathrm{~m}$

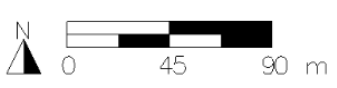

Outro detalhe interessante a se ressaltar relaciona-se aos maiores alcances obtidos nas camadas de $0,25-0,30$ e 0,30-0,35 m, nas quais foram verificados altos valores e continuidade da RP, provavelmente, em razão do intenso tráfego de máquinas/implementos que contribuiu para a formação de camadas compactadas de modo mais abrangente pela distribuição das pressões exercidas na superfície do solo (Freddi et al., 2007; Bergamin et al., 2010a; Bottega et al., 2011).

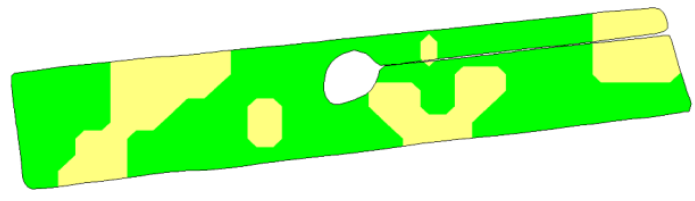

(B) $0,05-0,10 \mathrm{~m}$

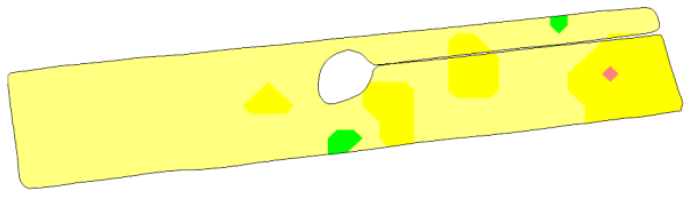

(D) $0,15-0,20 \mathrm{~m}$

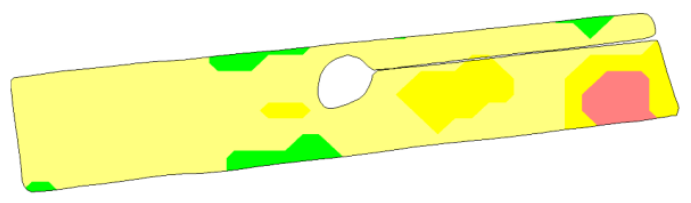

(F) $0,25-0,30 \mathrm{~m}$

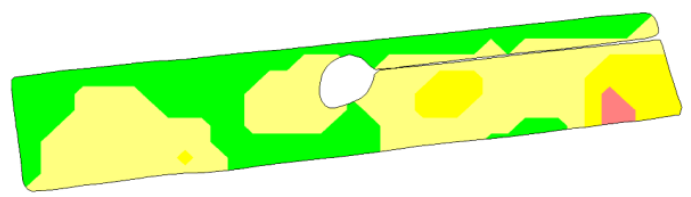

(H) $0,35-0,40 \mathrm{~m}$

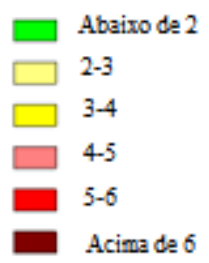

$\mathrm{MPa}$

Figura 1 - Mapas da resistência do solo à penetração (RP - MPa), utilizando o valor médio da camada: 0,00-0,05 (A); 0,05-0,10 (B); 0,10-0,15 (C); 0,15-0,20 (D); 0,20-0,25 (E); 0,25-0,30 (F); 0,30-0,35 (G); $0,35-0,40(\mathrm{H})$. Mapping of soil penetration resistance (RP - MPa) using the data average of depth: 0,00$-0,05 \mathrm{~m}(\mathrm{~A}) ; 0,05-0,10 \mathrm{~m}(\mathrm{~B}) ; 0,10-0,15 \mathrm{~m}(\mathrm{C}) ; 0,15-0,20 \mathrm{~m}$ (D); 0,20-0,25 m (E); 0,25-0,30 m (F); $0,30-0,35 \mathrm{~m}(\mathrm{G}) ; 0,35-0,40 \mathrm{~m}(\mathrm{H})$.

Nos mapas de isolinhas (Figura 1) com os valores médios de RP agrupados em 6 classes de cores, pode-se verificar a variabilidade da RP dentro de cada camada ao longo da área. Exceto nas camadas superficiais de 0-0,05 e 0,05-0,10 m e nas camadas profundas de 0,30-0,35 e 0,35-0,40 m, nas quais foi verificada maior concentração de áreas com RP inferior a 2 MPa e RP intermediária (2,00-3,00 MPa); nas demais, observou-se predominância de áreas com valores intermediários de RP (2,00-3,00 MPa), seguida de áreas com valores críticos de RP (3,00-4,00 MPa). Tais valores são considerados altos e acima do valor limitante abordado na literatura (Vaz et al., 2011; Betioli Júnior et al., 2012; Guimarães et al., 2013). 
Ainda analisando a Figura 1, observa-se que a RP aumenta da camada de 0,00-0,05 $\mathrm{m}$ até à camada de $0,25-0,30 \mathrm{~m}$. Comportamento semelhante foi verificado por Nagahama et al. (2016), quando avaliaram a variabilidade espacial da RP no perfil de um Argissolo Amarelo em sistemas de preparo do solo e velocidades de deslocamento do conjunto tratorequipamento; e por Coelho et al. (2012), ao avaliarem a variabilidade espacial da RP em área cultivada com manga, em Vertissolo, na região de Juazeiro-BA, e também por Cortez et al. (2014), quando analisaram RP em Argissolo Amarelo sob diferentes intensidades de tráfego de tratores.

$\mathrm{O}$ aumento da RP em profundidade até à camada de 0,25-0,30 m, provavelmente, ocorreu devido a um conjunto de fatores, entre os quais maior deposição de matéria orgânica e atividade biológica nas camadas superficiais que diminui a densidade do solo (Cortez et al., 2014; Nagahama et al., 2016), além do intenso tráfego máquinas/implementos em área sem revolvimento do solo, que favorece a distribuição de pressões resultantes do contanto solo-rodado, contribuindo para formação de camadas compactadas e para o aumento da RP. Oliveira et al. (2015), avaliando 0 efeito de manejos do solo sobre a RP, verificaram resultados semelhantes aos deste estudo.

Na Figura 2, são apresentados os mapas de isolinhas para resistência do solo à penetração média e máxima no perfil e profundidade da resistência máxima no perfil.
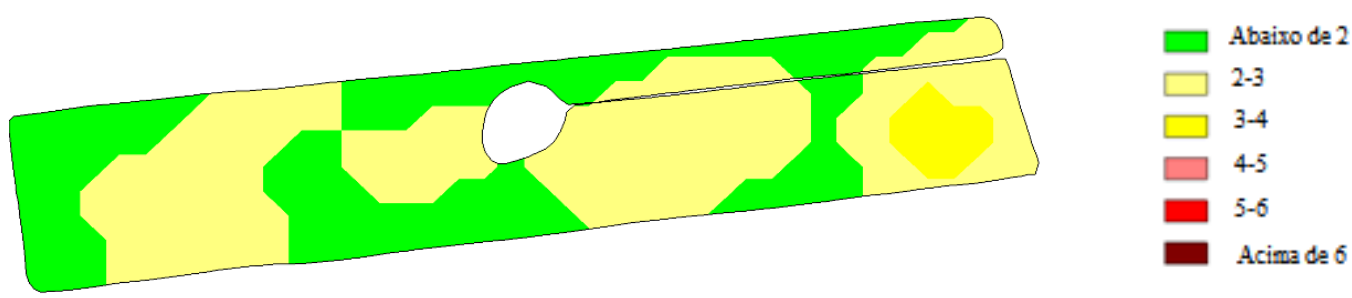

$\mathrm{MPa}$

(A) Resistência média $(0,00-0,40 \mathrm{~m})$
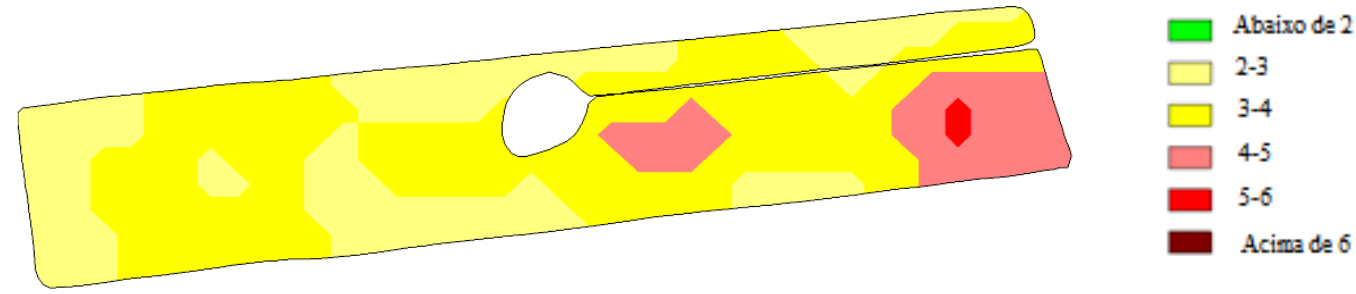

$\mathrm{MPa}$

(B) Resistência máxima $(0,00-0,40 \mathrm{~m})$
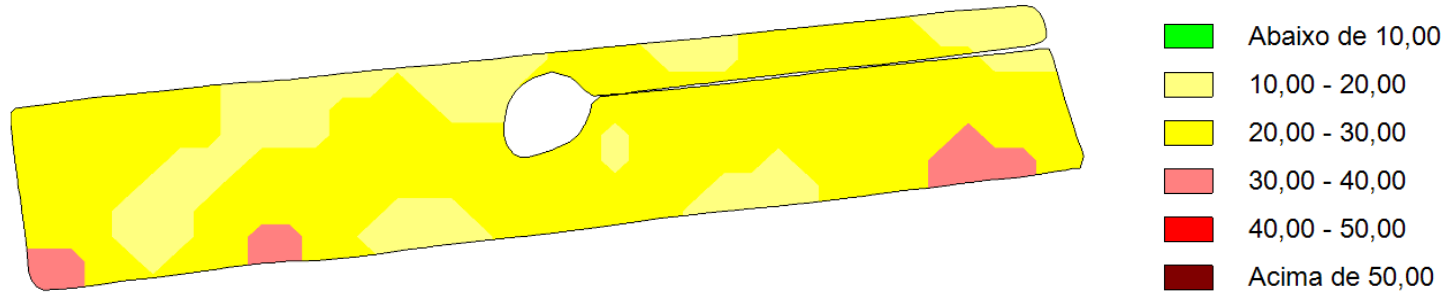

$\mathrm{cm}$

(C) Profundidade de RP máxima

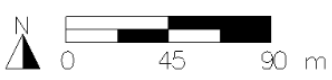

Figura 2 - Resistência do solo à penetração média (MPa) do perfil (A), resistência máxima (MPa) no perfil (B) e profundidade $(\mathrm{cm})$ da resistência máxima no perfil $(\mathrm{C})$. Soil penetration resistance avarage $(\mathrm{MPa})$ of profile $(\mathrm{A})$, resistence maximum in profile $(\mathrm{B})$ and depth $(\mathrm{cm})$ of resistence maximum in profile $(\mathrm{C})$.

Quanto à resistência média (Figura 2A), observa-se maior concentração de valores inferiores a $2 \mathrm{MPa}$, seguido da classe de valores intermediários de 2 a $3 \mathrm{MPa}$ e de valores críticos compreendidos entre 3 e $4 \mathrm{MPa}$. Embora estes valores se concentrem em área pouco representativa, e culturas como a soja não sejam tão sensíveis à faixa crítica (Marasca et al., 2011; Valadão et al., 2015), faz-se necessário um ma- nejo diferenciado para a remoção das camadas compactadas, uma vez que esta condição pode restringir o desenvolvimento radicular do milho (Freddi et al., 2007; Bergamin et al., 2010b), quando da sucessão com a soja.

Observa-se discrepância nos valores referentes à resistência máxima do perfil (Figura 2B), em que praticamente toda a área está ocupada com valores 
entre 2,00-6,00 MPa, com pontos intermediários e críticos em grande parte de sua extensão. No entanto, quando se considera sua resistência máxima, observam-se, por consequência, as profundidades máximas onde ocorrem tais resistências em seu perfil. Nas camadas de 0,10-0,20 m e 0,20-0,30 m, têm-se os níveis baixos $(<2,00 \mathrm{MPa})$ e intermediários de compactação $(2,00-3,00 \mathrm{MPa})$, respectivamente, seguido de níveis críticos de (3,00-4,00 MPa) e (4,00$-5,00 \mathrm{MPa}$ ) nas camadas de $0,20-0,30$ e 0,30-0,40 m, respectivamente. Porém, esta última camada está localizada na cabeceira, o que implica fatores externos e por isso a sua ocorrência.

Partindo da premissa de que um manejo diferenciado deve ser realizado dentro da área avaliada, ao analisar a Tabela 5 , verifica-se que, nas camadas de 0-0,05 m e 0,05-0,10 m, têm-se uma elevada por- centagem da área ocupada $(<2,00 \mathrm{MPa})$, ou seja, o percentual da área com problemas de compactação nessas profundidades é baixo. Para os níveis entre 2,00-3,00 MPa, pode-se observar um aumento na porcentagem em todas as camadas estudadas, exceto para a camada de 0,0-0,05 m, que não apresentou índice, indicando um aumento da RP gradualmente, de acordo com sua profundidade, consequentemente para os níveis 4,00-5,00 MPa, com um aumento gradativo da porcentagem em cada classe da RP, considerando a média da camada. Ao comparar o valor da RP média do perfil $(0,00-0,40 \mathrm{~m})$ com PM (Pressão máxima no perfil), verifica-se que a média mascara os resultados, uma vez que a área apresenta valores de RP máxima elevados, e que $11,83 \%$ da área tem pontos com RP entre 4 e $5 \mathrm{MPa}$, necessitando de um processo de remoção da compactação.

Tabela 5 - Porcentagem (\%) de área ocupada em cada classe da resistência à penetração. Percentage (\%) of área occupation in each class of penetration resistance.

\begin{tabular}{crccccc}
\hline \multirow{2}{*}{$\begin{array}{c}\text { Camadas } \\
(\mathrm{m})\end{array}$} & $<2,00$ & 2,00 a 3,00 & 3,00 a 4,00 & 4,00 a 5,00 & 5,00 a 6,00 & $>6,00$ \\
\cline { 2 - 7 } & 100 & - & - & - & - & - \\
$0,00-0,05$ & 67,18 & 32,82 & - & - & - & - \\
$0,05-0,10$ & 11,83 & 84,35 & 3,82 & - & - & - \\
$0,10-0,15$ & 1,15 & 78,63 & 19,85 & 0,38 & - & - \\
$0,15-0,20$ & 3,82 & 72,90 & 19,08 & 4,20 & - & - \\
$0,20-0,25$ & 5,34 & 75,57 & 14,89 & 4,20 & - & - \\
$0,25-0,30$ & 20,61 & 66,79 & 9,16 & 3,44 & - & - \\
$0,30-0,35$ & 37,02 & 50,76 & 11,07 & 1,15 & - & - \\
$0,35-0,40$ & 39,69 & 56,11 & 4,20 & - & - & - \\
$0,00-0,40$ & 0,00 & 33,59 & 53,82 & 11,83 & 0,76 & - \\
PM & & &
\end{tabular}

Avaliando a porcentagem de área ocupada pelos valores máximos de RP (Tabela 6), nota-se que, na camada $<10 \mathrm{~cm}$ e $>40 \mathrm{~cm}$, teve $0,0 \%$ ocupada, ou seja, não houve indícios de compactação. Entretanto, nas demais camadas, obtiveram-se índices de compactação, sendo a camada de 0,20-0,30 m com um valor maior de $70,23 \%$, a camada de $0,10-0,20 \mathrm{~m}$ apresentou um valor de $24,81 \%$, seguido da camada de 0,30-0,40 m com um valor de 4,9\%. Assim, em 95,04\% da área, a descompactação deveria ser feita na camada de 0,10-0,30 m, e apenas 4,96\% na de $0,30-0,40 \mathrm{~m}$.

Tabela 6 - Porcentagem de área ocupada pelos valores máximos de (RP) em cada classe. Percentage (\%) of área occupation by the values maximum of $(R P)$ in each class.

\begin{tabular}{cc}
\hline Profundidade da RP máxima & Porcentagem (\%) \\
\hline$<10 \mathrm{~cm}$ & 0,00 \\
$10 \mathrm{a} 20 \mathrm{~cm}$ & 24,81 \\
$20 \mathrm{a} 30 \mathrm{~cm}$ & 70,23 \\
$30 \mathrm{a} 40 \mathrm{~cm}$ & 4,96 \\
$>40 \mathrm{~cm}$ & 0,0 \\
\hline
\end{tabular}

\section{Conclusões}

A resistência do solo à penetração apresenta dependência espacial em todas as camadas dentro do gride amostral, sendo os maiores alcances nas camadas de 0,25-0,30 m e 0,30$-0,35 \mathrm{~m}$, nas quais foram verificados níveis críticos, provavelmente, em razão do contínuo tráfego de máquinas/implementos.

Há incremento da resistência do solo à penetração com 0 aumento da profundidade, atingindo níveis máximos principalmente entre 0,20 e $0,25 \mathrm{~m}$.

A adoção do sistema de semeadura direta $e$ o constante tráfego de máquinas na área favorecem o aparecimento de valores intermediários (2,00-3,00 $\mathrm{MPa})$ e críticos (3,00-4,00 MPa) de resistência à penetração.

As técnicas geoestatísticas permitem identificar o zoneamento da área, definindo regiões com níveis críticos de compactação, auxiliando, assim, na tomada de decisões quanto a procedimentos de manejo de solo 


\section{Referências}

Albiero D, Maciel AJS, Milan M, Monteiro LA, Mion RL (2012) Avaliação da distribuição de sementes por uma semeadora de anel interno rotativo utilizando média móvel exponencial. Revista Ciência Agronômica 43(1):86-95.

Alves SMF, Queiroz DM, Alcântara GR, Reis EF (2014) Variabilidade espacial de atributos físicoquímicos do solo usando técnicas de análise de componentes principais e geoestatística. Bioscience Journal 30(3):22-30.

Bergamin AC, Vitorino ACT, Franchini JC, Souza CMA, Souza FR (2010a) Compactação em um Latossolo Vermelho Distroférrico e suas relações com o crescimento radicular do milho. Revista Brasileira de Ciência do Solo 34(3):681-691.

Bergamin AC, Vitorino ACT, Lempp B, Souza CMA, Souza FR (2010b) Anatomia radicular de milho em solo compactado. Pesquisa agropecuária Brasileira 45(3):299-305.

Betioli Júnior E, Moreira WH, Tormena CA, Ferreira CJB, Silva AP, Giarola NFB (2012) Intervalo hídrico ótimo e grau de compactação de um Latossolo Vermelho após 30 anos sob plantio direto. Revista Brasileira de Ciência do Solo 36(3):971-982.

Bottega EL, Bottega SP, Silva AS, Queiroz DM, Souza CMA, Rafull LZL (2011) Variabilidade espacial da resistência do solo à penetração em um Latossolo Vermelho distroférrico. Revista Brasileira de Ciências Agrárias 6(2):331-336.

Bottega EL, Queiroz DM, Pinto FAC, Souza CMA (2013) Variabilidade espacial de atributos do solo em sistema de semeadura direta com rotação de culturas no cerrado brasileiro. Revista Ciência Agronômica 44(1):1-9.

Coelho DS, Cortez JW, Olszevski N (2012) Variabilidade espacial da resistência mecânica à penetração em Vertissolo cultivado com manga no perímetro irrigado de Mandacaru, Juazeiro, Bahia, Brasil. Revista Brasileira de Ciência do Solo 36(3):755-763.

Cortez JW, Olszevski N, Pimenta WA, Patrocínio Filho, AP, Souza EB, Nagahama HJ (2014) Avaliação da intensidade de tráfego de tratores em alguns atributos físicos de um Argissolo Amarelo. Revista Brasileira de Ciência do Solo 38(3):1000-1010.

Freddi OS, Centurion JF, Beutler NA, Aratani RG, Leonel CL (2007) Compactação do solo no crescimento radicular e produtividade da cultura do milho. Revista Brasileira de Ciência do Solo 31(4):627-636.

Guimarães RML, Blainski E, Fidalski J (2013) Intervalo hídrico ótimo para avaliação da degradação física do solo. Revista Brasileira de Ciência do Solo 37(6):1512-1521.
Marasca I, Oliveira CAA, Guimarães EC, Cunha JPAR, Assis RL, Perin A, Menezes LAS (2011) Variabilidade espacial da resistência do solo à penetração e teor de agua em sistema de plantio direto na cultura da soja. Bioscience Journal 27(2):239-246.

Melo RP, Albiero D, Monteiro LA, Souza FH, Silva JG (2013) Qualidade na distribuição de sementes de milho em semeadoras em um solo cearense. Revista Ciência Agronômica.44(1):94-101.

Nagahama HJ, Cortez JW, Pimenta WA, Pereira Filho AP, Souza EB (2016) Resistência do solo à penetração em sistemas de preparo e velocidades de deslocamento do trator. Comunicata Scientiae 7(1):56-65.

Oliveira DMS, Lima RP, Verburg EEJ (2015) Qualidade física do solo sob diferentes sistemas de manejo e aplicação de dejeto líquido suíno. Revista Brasileira de Engenharia Agrícola e Ambiental 19 (3):280-285.

Oliveira Filho FX, Miranda NO, Medeiros JF, Silva PCM, Mesquita FO, Costa TKG (2015) Zona de manejo para preparo do solo na cultura da cana-deaçúcar. Revista Brasileira de Engenharia Agrícola e Ambiental 19(2):186-193.

Oliveira IA, Campos MCC, Soares MPR, Aquino RE, Marques Júnior J, Nascimento EP (2013) Variabilidade espacial de atributos físicos em um Cambissolo Háplico sob diferentes usos na região sul do Amazonas. Revista Brasileira de Ciência do Solo 37(4):1103-1112.

Rosolem CA, Fernandez EM, Andreottl M, Crusciol CAC (1999) Crescimento radicular de plântulas de milho afetado pela resistência do solo à penetração. Pesquisa Agropecuária Brasileira 34(5):821-828.

Silva RP, Cassia MT, Voltarelli MA, Compagnon AM, Furlani CEAF (2013) Qualidade da colheita mecanizada de feijão em dois sistemas de preparo do solo. Revista Ciência Agronômica 44(1): 61-69.

Streck CA, Reinert DJ, Reichert JM, Kaiser DR (2004) Modificações em propriedades físicas com a compactação do solo causada pelo tráfego induzido de um trator em plantio direto. Ciência Rural 34(3):755-760.

Valadão FCA, Weber OL, Valadão Júnior DD, Scarpinelli A, Deina FR, Bianchini A (2015) Adubação fosfatada e compactação do solo: sistema radicular da soja e do milho e atributos físicos do solo. Revista Brasileira de Ciência do Solo 39(1):243-255.

Vaz CMP, Manieri JM, Maria IC, Tuller M (2011) Modeling and correction of soil penetration resistance for varying soil water content. Geoderma 166 (2):92-101. 